\section{Crystal structure of 9-butyl-6-[2-(pyridin- 4-yl)ethenyl]carbazol-3-amine}

\author{
Ping Zhang, ${ }^{a}$ Xiang-Yang Bai ${ }^{\mathrm{b}}$ and Ting Zhang ${ }^{\mathrm{a} *}$

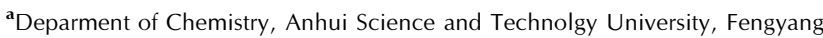 \\ 233100, People's Republic of China, and ${ }^{\mathbf{b}}$ College of Chemistry \& Chemical \\ Engineering, Anhui University, Hefei 230039, People's Republic of China, Key \\ Laboratory of Functional Inorganic Materials of Anhui Province, Hefei 230039, \\ People's Republic of China. *Correspondence e-mail: zhangt@ahstu.edu.cn \\ Received 25 March 2015; accepted 22 April 2015
}

Edited by W. T. A. Harrison, University of Aberdeen, Scotland

The asymmetric unit of the title compound, $\mathrm{C}_{23} \mathrm{H}_{23} \mathrm{~N}_{3}$, consists of two molecules, $A$ and $B$, with different conformations. In molecule $A$, the dihedral angle between the carbazole ring system (r.m.s. deviation $=0.028 \AA$ ) and the pyridine ring is $20.28(9)^{\circ}$ and the $\mathrm{N}-\mathrm{C}-\mathrm{C}-\mathrm{C}$ torsion angle of the butyl side chain is $-63.4(3)^{\circ}$. The equivalent data for molecule $B$ are $0.065 \AA, 48.28(11)^{\circ}$ and $61.0(3)^{\circ}$, respectively. In the crystal, the components are connected by weak $\mathrm{N}-\mathrm{H} \cdots \mathrm{N}$ hydrogen bonds, generating [030] $C(14)$ chains of alternating $A$ and $B$ molecules.

Keywords: crystal structure; carbazol-3-amine; hydrogen bonding.

CCDC reference: 1061103

\section{Related literature}

For background to the applications of carbazoles, see: Wang et al. (2013); Feng et al. (2013); Park et al. (2015). For further synthetic details, see: Zhang et al. (2014).

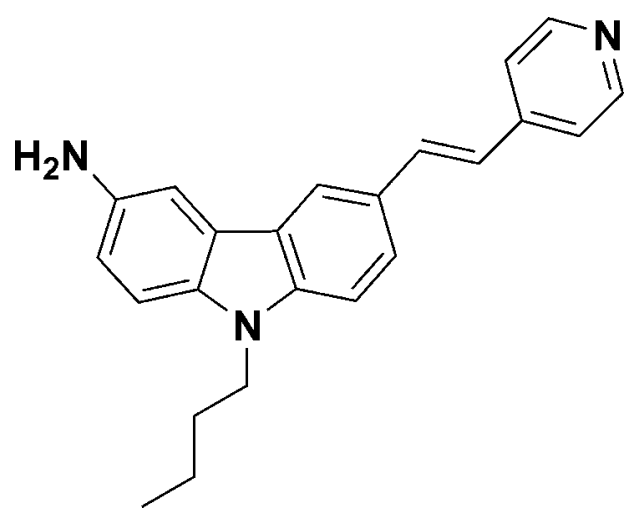

2. Experimental

2.1. Crystal data

$\mathrm{C}_{23} \mathrm{H}_{23} \mathrm{~N}_{3}$

$M_{r}=341.44$

Monoclinic, $P 2_{1} / c$

$a=11.296$ (4) А

$b=18.719$ (7) $\AA$

$c=17.829(7) \AA$

$\beta=95.362(5)^{\circ}$

$$
\begin{aligned}
& V=3753(2) \AA^{3} \\
& Z=8 \\
& \text { Mo } K \alpha \text { radiation } \\
& \mu=0.07 \mathrm{~mm}^{-1} \\
& T=296 \mathrm{~K} \\
& 0.30 \times 0.20 \times 0.20 \mathrm{~mm}
\end{aligned}
$$

\subsection{Data collection}

Bruker SMART CCD

diffractometer

26552 measured reflections

6604 independent reflections 4780 reflections with $I>2 \sigma(I)$ $R_{\text {int }}=0.029$

\subsection{Refinement}

$R\left[F^{2}>2 \sigma\left(F^{2}\right)\right]=0.053$

$w R\left(F^{2}\right)=0.166$

$S=1.14$

6604 reflections

471 parameters

5 restraints

$\mathrm{H}$-atom parameters constrained

$\Delta \rho_{\max }=0.66 \mathrm{e} \AA^{-3}$

$\Delta \rho_{\min }=-0.28$ e $\AA^{-3}$

Table 1

Hydrogen-bond geometry $\left(\AA{ }^{\circ}\right)$.

\begin{tabular}{lllll}
\hline$D-\mathrm{H} \cdots A$ & $D-\mathrm{H}$ & $\mathrm{H} \cdots A$ & $D \cdots A$ & $D-\mathrm{H} \cdots A$ \\
\hline $\mathrm{N} 2-\mathrm{H} 2 D \cdots \mathrm{N} 6$ & 0.86 & 2.56 & $3.154(3)$ & 128 \\
$\mathrm{~N} 5-\mathrm{H} 5 B \cdots \mathrm{N} 3^{\mathrm{i}}$ & 0.86 & 2.36 & $3.163(3)$ & 156 \\
\hline
\end{tabular}

Symmetry code: (i) $-x+1, y+\frac{3}{2},-z+\frac{1}{2}$.

Data collection: SMART (Bruker, 2007); cell refinement: SAINT (Bruker, 2007); data reduction: $S A I N T$; $\operatorname{program}(\mathrm{s})$ used to solve structure: SHELXS97 (Sheldrick, 2008); program(s) used to refine structure: SHELXL97 (Sheldrick, 2008); molecular graphics: SHELXTL (Sheldrick, 2008); software used to prepare material for publication: SHELXTL.

\section{Acknowledgements}

We gratefully acknowledge the Natural Science Foundation of Anhui Province (grant No. 1508085QB41) for supporting this study. 


\section{data reports}

Supporting information for this paper is available from the IUCr electronic archives (Reference: HB7392).

\section{References}

Bruker (2007). SMART and SAINT. Bruker AXS Inc., Madison, Wisconsin, USA.
Feng, X. J., Tian, P. Z., Xu, Z., Chen, S. F. \& Wong, M. S. (2013). J. Org. Chem. 78, $11318-11325$.

Park, Y. I., Postupna, O., Zhugayevych, A., Shin, H., Park, Y. S., Kim, B., Yen, H. J., Cheruku, P., Martinez, J. S., Park, J. W., Tretiak, S. \& Wang, H. L. (2015). Chem. Sci. 6, 789-797.

Sheldrick, G. M. (2008). Acta Cryst. A64, 112-122.

Wang, H. W., Chen, Y., Ye, W. B., Xu, J. K., Liu, D. F., Yang, J. X., Kong, L., Zhou, H. P., Tian, Y. P. \& Tao, X. T. (2013). Dyes Pigments, 96, 738-747.

Zhang, P., Liu, J., Huang, J. Y. \& Yang, J. X. (2014). Chin. J. Appl. Chem. 31, 1171-1176. 


\section{supporting information}

Acta Cryst. (2015). E71, o345-o346 [https://doi.org/10.1107/S2056989015007975]

\section{Crystal structure of 9-butyl-6-[2-(pyridin-4-yl)ethenyl]carbazol-3-amine}

\section{Ping Zhang, Xiang-Yang Bai and Ting Zhang}

\section{S1. Comment}

The title compound is a carbazole derivative with an amine group and a pyridine group. Carbazole is usually utilized in organic functional materials due to it is a large conjugated system with prominent hole-transporting(Wang et al., 2013). The pyridine group havebeen used as heavy metal sensors (Feng et al., 2013) and the amino groupcan be regulated by acid-base based on intermolecular charge transfer(Park et al., 2015). The title compound might be able to get multiple application fields.

In (I) (Fig.1),

The bond distances of $\mathrm{C} 15-\mathrm{N} 2$ is not equal to the bond distance of $\mathrm{C} 31-\mathrm{N} 5$, which are $1.403 \AA$ and $1.395 \AA$. The torsion angle of $\mathrm{C} 8-\mathrm{C} 17-\mathrm{C} 18-\mathrm{C} 19$ are similiar to $\mathrm{C} 36-\mathrm{C} 40-\mathrm{C} 41-\mathrm{C} 42$, but the dihedral angles of the phenyl group and pyridine group is different in the two molecular, which are $18.97^{\circ}$ and $45.44^{\circ}$, respectively.

The crystal packing shows that the related molecules are linking by N6 $\cdots \mathrm{H} 2 \mathrm{D}-\mathrm{N} 2, \mathrm{~N} 5 \cdots \mathrm{H} 16-\mathrm{C} 16$ hydrogen bonds.

\section{S2. Refinement}

All hydrogen atoms were placed in geometrically idealized positions and constrained to ride on their parent atoms, with C $-\mathrm{H}=0.93 \AA$ and $U_{\text {iso }}(\mathrm{H})=1.2 U_{\text {eq }}$.
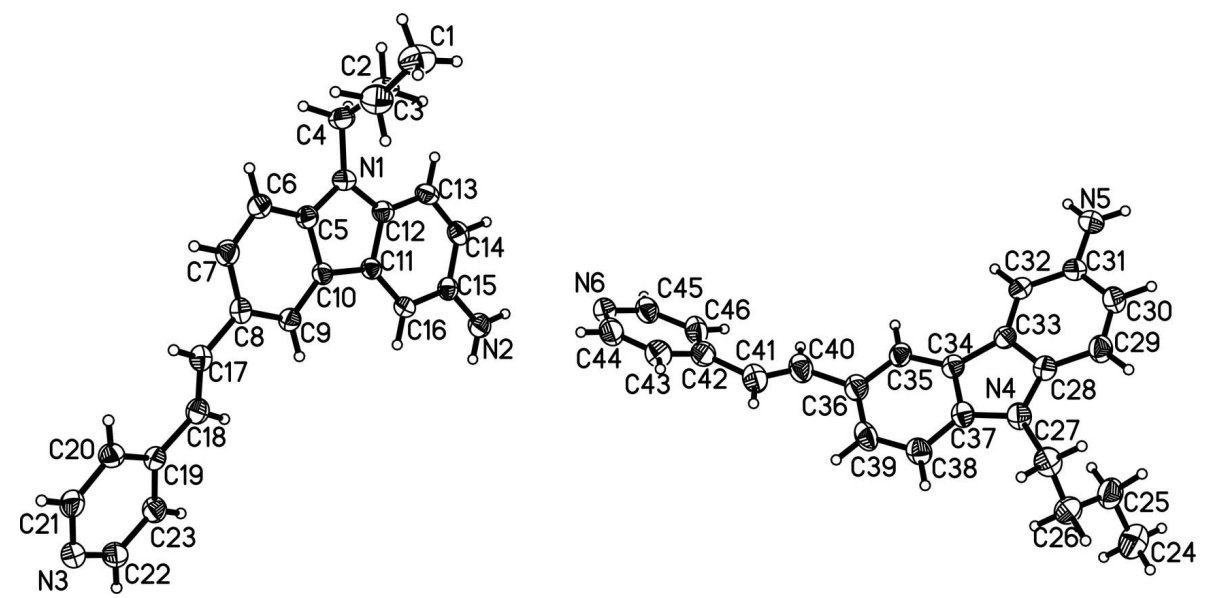

Figure 1

The molecular structure of the title compound. 


\section{9-Butyl-6-[2-(pyridin-4-yl)ethenyl]carbazol-3-amine}

Crystal data

$$
\begin{aligned}
& \mathrm{C}_{23} \mathrm{H}_{23} \mathrm{~N}_{3} \\
& M_{r}=341.44 \\
& \text { Monoclinic, } P 2_{1} / c \\
& a=11.296(4) \AA \\
& b=18.719(7) \AA \\
& c=17.829(7) \AA \\
& \beta=95.362(5)^{\circ} \\
& V=3753(2) \AA^{3} \\
& Z=8
\end{aligned}
$$

\section{Data collection}

Bruker SMART CCD diffractometer

Radiation source: fine-focus sealed tube Graphite monochromator $\omega$ scans

26552 measured reflections

6604 independent reflections

\section{Refinement}

Refinement on $F^{2}$

Least-squares matrix: full

$R\left[F^{2}>2 \sigma\left(F^{2}\right)\right]=0.053$

$w R\left(F^{2}\right)=0.166$

$S=1.14$

6604 reflections

471 parameters

5 restraints

Primary atom site location: structure-invariant direct methods
$F(000)=1456$

$D_{\mathrm{x}}=1.209 \mathrm{Mg} \mathrm{m}^{-3}$

Mo $K \alpha$ radiation, $\lambda=0.71073 \AA$

Cell parameters from 7036 reflections

$\theta=2.3-24.3^{\circ}$

$\mu=0.07 \mathrm{~mm}^{-1}$

$T=296 \mathrm{~K}$

$0.30 \times 0.20 \times 0.20 \mathrm{~mm}$

4780 reflections with $I>2 \sigma(I)$

$R_{\text {int }}=0.029$

$\theta_{\text {max }}=25.0^{\circ}, \theta_{\text {min }}=1.6^{\circ}$

$h=-13 \rightarrow 12$

$k=-20 \rightarrow 22$

$l=-21 \rightarrow 21$

Secondary atom site location: difference Fourier map

Hydrogen site location: inferred from neighbouring sites

$\mathrm{H}$-atom parameters constrained

$w=1 /\left[\sigma^{2}\left(F_{\mathrm{o}}^{2}\right)+(0.0773 P)^{2}+0.9436 P\right]$

where $P=\left(F_{\mathrm{o}}{ }^{2}+2 F_{\mathrm{c}}{ }^{2}\right) / 3$

$(\Delta / \sigma)_{\max }<0.001$

$\Delta \rho_{\max }=0.66 \mathrm{e} \AA^{-3}$

$\Delta \rho_{\min }=-0.28$ e $\AA^{-3}$

Special details

Geometry. All e.s.d.'s (except the e.s.d. in the dihedral angle between two 1.s. planes) are estimated using the full covariance matrix. The cell e.s.d.'s are taken into account individually in the estimation of e.s.d.'s in distances, angles and torsion angles; correlations between e.s.d.'s in cell parameters are only used when they are defined by crystal symmetry. An approximate (isotropic) treatment of cell e.s.d.'s is used for estimating e.s.d.'s involving 1.s. planes.

Refinement. Refinement of $F^{2}$ against ALL reflections. The weighted $R$-factor $w R$ and goodness of fit $S$ are based on $F^{2}$, conventional $R$-factors $R$ are based on $F$, with $F$ set to zero for negative $F^{2}$. The threshold expression of $F^{2}>\sigma\left(F^{2}\right)$ is used only for calculating $R$-factors(gt) etc. and is not relevant to the choice of reflections for refinement. $R$-factors based on $F^{2}$ are statistically about twice as large as those based on $F$, and $R$ - factors based on ALL data will be even larger.

Fractional atomic coordinates and isotropic or equivalent isotropic displacement parameters $\left(\AA^{2}\right)$

\begin{tabular}{lllll}
\hline & $x$ & $y$ & $z$ & $U_{\text {iso }} * / U_{\text {eq }}$ \\
\hline C8 & $0.1402(2)$ & $0.18126(11)$ & $0.33640(12)$ & $0.0506(5)$ \\
C10 & $0.07484(18)$ & $0.28290(11)$ & $0.26009(11)$ & $0.0439(5)$ \\
C11 & $0.06577(18)$ & $0.33974(11)$ & $0.20495(11)$ & $0.0434(5)$ \\
C18 & $0.3040(2)$ & $0.09517(12)$ & $0.31670(12)$ & $0.0526(6)$ \\
H18 & 0.3148 & 0.1176 & 0.2713 & $0.063 *$ \\
C17 & $0.2208(2)$ & $0.12164(12)$ & $0.35622(13)$ & $0.0526(5)$
\end{tabular}




\begin{tabular}{|c|c|c|c|c|}
\hline H17 & 0.2125 & 0.0997 & 0.4022 & $0.063 *$ \\
\hline $\mathrm{C} 12$ & $-0.04171(18)$ & $0.37514(11)$ & $0.21275(11)$ & $0.0457(5)$ \\
\hline $\mathrm{C} 16$ & 0.14005 (19) & $0.36372(11)$ & $0.15188(12)$ & $0.0471(5)$ \\
\hline H16 & 0.2116 & 0.3405 & 0.1465 & $0.057^{*}$ \\
\hline $\mathrm{C} 15$ & $0.10675(19)$ & $0.42226(11)$ & $0.10720(12)$ & $0.0470(5)$ \\
\hline $\mathrm{C} 5$ & $-0.02863(19)$ & $0.28735(11)$ & $0.29917(12)$ & $0.0488(5)$ \\
\hline C9 & $0.15800(19)$ & $0.22968(11)$ & $0.27827(12)$ & $0.0492(5)$ \\
\hline H9 & 0.2252 & 0.2260 & 0.2521 & $0.059^{*}$ \\
\hline C19 & $0.38121(18)$ & 0.03355 (11) & $0.33784(12)$ & $0.0464(5)$ \\
\hline $\mathrm{C} 7$ & $0.0385(2)$ & $0.18909(13)$ & $0.37517(13)$ & $0.0577(6)$ \\
\hline H7 & 0.0278 & 0.1578 & 0.4145 & $0.069 *$ \\
\hline $\mathrm{C} 13$ & $-0.0760(2)$ & $0.43368(12)$ & $0.16804(13)$ & $0.0526(5)$ \\
\hline H13 & -0.1474 & 0.4571 & 0.1732 & $0.063^{*}$ \\
\hline $\mathrm{C} 20$ & $0.3807(2)$ & $-0.00335(13)$ & $0.40535(13)$ & $0.0559(6)$ \\
\hline $\mathrm{H} 20$ & 0.3287 & 0.0102 & 0.4403 & $0.067^{*}$ \\
\hline $\mathrm{C} 34$ & $0.5999(2)$ & $1.02101(12)$ & $0.11976(11)$ & $0.0492(5)$ \\
\hline $\mathrm{C} 33$ & $0.57620(19)$ & $1.09595(12)$ & $0.12853(12)$ & $0.0482(5)$ \\
\hline $\mathrm{C} 14$ & $-0.0013(2)$ & $0.45628(12)$ & $0.11577(12)$ & $0.0520(5)$ \\
\hline H14 & -0.0236 & 0.4953 & 0.0854 & $0.062 *$ \\
\hline $\mathrm{C} 28$ & $0.6780(2)$ & $1.12522(12)$ & $0.16791(12)$ & $0.0525(5)$ \\
\hline $\mathrm{C} 21$ & $0.4568(2)$ & $-0.05970(13)$ & $0.42064(14)$ & $0.0608(6)$ \\
\hline $\mathrm{H} 21$ & 0.4538 & -0.0833 & 0.4663 & $0.073 *$ \\
\hline $\mathrm{C} 23$ & $0.4605(2)$ & $0.00853(13)$ & $0.28947(13)$ & $0.0568(6)$ \\
\hline $\mathrm{H} 23$ & 0.4644 & 0.0305 & 0.2430 & $0.068^{*}$ \\
\hline $\mathrm{C} 37$ & $0.7160(2)$ & $1.00873(12)$ & $0.15361(12)$ & $0.0532(6)$ \\
\hline $\mathrm{C} 35$ & $0.5315(2)$ & $0.96430(12)$ & $0.09044(13)$ & $0.0554(6)$ \\
\hline H35 & 0.4561 & 0.9729 & 0.0665 & $0.066^{*}$ \\
\hline $\mathrm{C} 36$ & $0.5740(2)$ & $0.89555(13)$ & $0.09647(14)$ & $0.0635(6)$ \\
\hline C6 & $-0.0456(2)$ & $0.24026(13)$ & $0.35797(13)$ & $0.0582(6)$ \\
\hline H6 & -0.1123 & 0.2437 & 0.3846 & $0.070^{*}$ \\
\hline $\mathrm{C} 31$ & $0.4804(2)$ & $1.21038(13)$ & $0.12857(15)$ & $0.0608(6)$ \\
\hline $\mathrm{C} 45$ & $0.2326(3)$ & $0.66774(13)$ & $-0.00112(15)$ & $0.0666(7)$ \\
\hline $\mathrm{H} 45$ & 0.1796 & 0.6703 & -0.0442 & $0.080^{*}$ \\
\hline $\mathrm{C} 4$ & $-0.2165(2)$ & $0.36238(13)$ & $0.28986(14)$ & $0.0601(6)$ \\
\hline $\mathrm{H} 4 \mathrm{~A}$ & -0.2499 & 0.3222 & 0.3150 & $0.072 *$ \\
\hline $\mathrm{H} 4 \mathrm{~B}$ & -0.2673 & 0.3715 & 0.2439 & $0.072 *$ \\
\hline C32 & $0.4770(2)$ & $1.13893(13)$ & $0.10920(13)$ & $0.0557(6)$ \\
\hline H32 & 0.4091 & 1.1196 & 0.0834 & $0.067^{*}$ \\
\hline $\mathrm{C} 22$ & $0.5335(2)$ & $-0.04822(13)$ & $0.30896(15)$ & $0.0630(6)$ \\
\hline $\mathrm{H} 22$ & 0.5850 & -0.0634 & 0.2745 & $0.076^{*}$ \\
\hline C39 & $0.6912(3)$ & $0.88488(14)$ & $0.12855(15)$ & $0.0710(7)$ \\
\hline H39 & 0.7213 & 0.8386 & 0.1311 & $0.085^{*}$ \\
\hline $\mathrm{C} 26$ & $0.9762(2)$ & $1.09499(16)$ & $0.16513(17)$ & $0.0767(8)$ \\
\hline $\mathrm{H} 26 \mathrm{~A}$ & 1.0532 & 1.0988 & 0.1939 & $0.092 *$ \\
\hline H26B & 0.9787 & 1.0538 & 0.1324 & $0.092 *$ \\
\hline $\mathrm{C} 42$ & $0.3969(2)$ & $0.71893(14)$ & $0.07683(16)$ & $0.0705(6)$ \\
\hline $\mathrm{C} 27$ & $0.8826(2)$ & $1.08206(15)$ & $0.21935(15)$ & $0.0713(7)$ \\
\hline H27A & 0.9045 & 1.0400 & 0.2493 & $0.086^{*}$ \\
\hline
\end{tabular}




\begin{tabular}{|c|c|c|c|c|}
\hline $\mathrm{H} 27 \mathrm{~B}$ & 0.8819 & 1.1224 & 0.2534 & $0.086^{*}$ \\
\hline $\mathrm{C} 41$ & $0.4903(3)$ & $0.77281(15)$ & $0.09790(17)$ & $0.0784(8)$ \\
\hline H41 & 0.5541 & 0.7593 & 0.1317 & $0.094 *$ \\
\hline $\mathrm{C} 40$ & 0.4888 & $0.83625(14)$ & $0.07270(16)$ & $0.0747(7)$ \\
\hline $\mathrm{H} 40$ & 0.4284 & 0.8473 & 0.0354 & $0.090 *$ \\
\hline $\mathrm{C} 3$ & $-0.2190(2)$ & $0.42683(15)$ & $0.33998(15)$ & $0.0681(7)$ \\
\hline $\mathrm{H} 3 \mathrm{~A}$ & -0.1855 & 0.4671 & 0.3150 & $0.082 *$ \\
\hline H3B & -0.3011 & 0.4383 & 0.3465 & $0.082 *$ \\
\hline C38 & $0.7640(2)$ & $0.94025(13)$ & $0.15659(14)$ & $0.0647(7)$ \\
\hline H38 & 0.8418 & 0.9319 & 0.1766 & $0.078^{*}$ \\
\hline $\mathrm{C} 29$ & $0.6807(2)$ & $1.19679(13)$ & $0.18829(14)$ & $0.0644(6)$ \\
\hline $\mathrm{H} 29$ & 0.7480 & 1.2164 & 0.2146 & $0.077 *$ \\
\hline $\mathrm{C} 46$ & $0.3153(3)$ & $0.72233(14)$ & $0.01270(15)$ & $0.0739(7)$ \\
\hline H46 & 0.3164 & 0.7607 & -0.0203 & $0.089 *$ \\
\hline $\mathrm{C} 30$ & $0.5828(2)$ & $1.23771(14)$ & $0.16907(15)$ & $0.0659(7)$ \\
\hline H30 & 0.5840 & 1.2855 & 0.1833 & $0.079 *$ \\
\hline $\mathrm{C} 44$ & $0.3030(3)$ & $0.61135(15)$ & $0.10490(18)$ & $0.0750(8)$ \\
\hline H44 & 0.2991 & 0.5731 & 0.1378 & $0.090 *$ \\
\hline $\mathrm{C} 43$ & $0.3871(3)$ & $0.66132(15)$ & $0.12302(17)$ & $0.0759(7)$ \\
\hline H43 & 0.4381 & 0.6566 & 0.1668 & $0.091 *$ \\
\hline $\mathrm{C} 2$ & -0.1533 & $0.41786(17)$ & $0.41516(16)$ & $0.0815(8)$ \\
\hline $\mathrm{H} 2 \mathrm{~A}$ & -0.0691 & 0.4136 & 0.4093 & $0.098^{*}$ \\
\hline $\mathrm{H} 2 \mathrm{~B}$ & -0.1787 & 0.3739 & 0.4375 & $0.098^{*}$ \\
\hline $\mathrm{C} 25$ & $0.9563(3)$ & $1.16126(16)$ & $0.11666(18)$ & $0.0816(8)$ \\
\hline $\mathrm{H} 25 \mathrm{~A}$ & 0.8837 & 1.1552 & 0.0837 & $0.098 *$ \\
\hline $\mathrm{H} 25 \mathrm{~B}$ & 0.9451 & 1.2018 & 0.1491 & $0.098 *$ \\
\hline $\mathrm{C} 1$ & $-0.1724(3)$ & 0.48013 (19) & $0.46861(17)$ & $0.0927(10)$ \\
\hline H1A & -0.1630 & 0.5245 & 0.4429 & $0.139 *$ \\
\hline H1B & -0.1150 & 0.4774 & 0.5118 & $0.139 *$ \\
\hline $\mathrm{H} 1 \mathrm{C}$ & -0.2511 & 0.4775 & 0.4846 & $0.139 *$ \\
\hline $\mathrm{C} 24$ & $1.0568(3)$ & $1.1780(2)$ & $0.0689(2)$ & $0.1101(12)$ \\
\hline $\mathrm{H} 24 \mathrm{~A}$ & 1.1303 & 1.1805 & 0.1005 & $0.165^{*}$ \\
\hline $\mathrm{H} 24 \mathrm{~B}$ & 1.0419 & 1.2229 & 0.0439 & $0.165^{*}$ \\
\hline $\mathrm{H} 24 \mathrm{C}$ & 1.0620 & 1.1410 & 0.0320 & $0.165^{*}$ \\
\hline N2 & $0.17911(18)$ & $0.44634(10)$ & $0.05257(10)$ & $0.0597(5)$ \\
\hline $\mathrm{H} 2 \mathrm{C}$ & 0.2448 & 0.4247 & 0.0466 & $0.072 *$ \\
\hline $\mathrm{H} 2 \mathrm{D}$ & 0.1578 & 0.4827 & 0.0251 & $0.072 *$ \\
\hline N1 & $-0.09916(16)$ & $0.34248(10)$ & $0.26974(10)$ & $0.0521(5)$ \\
\hline N4 & $0.76339(18)$ & $1.07204(10)$ & $0.18222(11)$ & $0.0597(5)$ \\
\hline N3 & $0.53538(18)$ & -0.08319 & $0.37428(12)$ & $0.0618(5)$ \\
\hline N6 & $0.2259(2)$ & $0.61214(11)$ & $0.04444(13)$ & $0.0665(6)$ \\
\hline N5 & 0.38357 (19) & $1.25534(13)$ & $0.11005(17)$ & $0.0895(8)$ \\
\hline H5A & 0.3199 & 1.2387 & 0.0860 & $0.107 *$ \\
\hline H5B & 0.3875 & 1.2997 & 0.1227 & $0.107 *$ \\
\hline
\end{tabular}


Atomic displacement parameters $\left(\AA^{2}\right)$

\begin{tabular}{|c|c|c|c|c|c|c|}
\hline & $U^{11}$ & $U^{22}$ & $U^{33}$ & $U^{12}$ & $U^{13}$ & $U^{23}$ \\
\hline $\mathrm{C} 8$ & 0.0577 (14) & $0.0424(12)$ & $0.0510(12)$ & $-0.0030(10)$ & $0.0014(10)$ & $0.0007(10)$ \\
\hline $\mathrm{C} 10$ & $0.0447(12)$ & $0.0384(11)$ & $0.0484(11)$ & $-0.0009(9)$ & $0.0029(9)$ & -0.0021 \\
\hline $\mathrm{C} 11$ & 0.0433 (11) & $0.0396(11)$ & $0.0469(11)$ & $0.0006(9)$ & $0.0023(9)$ & $-0.0047(9)$ \\
\hline C18 & $0.0575(14)$ & $0.0531(13)$ & $0.0472(12)$ & $-0.0038(11)$ & $0.0055(10)$ & $0.0053(10)$ \\
\hline $\mathrm{C} 17$ & $0.0592(14)$ & 0.0469 (12) & $0.0517(12)$ & $-0.0023(11)$ & $0.0052(11)$ & $0.0043(10)$ \\
\hline $\mathrm{C} 12$ & $0.0450(12)$ & $0.0457(12)$ & $0.0463(11)$ & $-0.0003(10)$ & $0.0034(9)$ & $-0.0060(9)$ \\
\hline $\mathrm{C} 16$ & $0.0465(12)$ & $0.0435(12)$ & $0.0521(12)$ & $0.0041(9)$ & $0.0084(10)$ & $-0.0023(10)$ \\
\hline $\mathrm{C} 15$ & $0.0537(13)$ & $0.0432(12)$ & $0.0442(11)$ & $-0.0033(10)$ & $0.0039(10)$ & $-0.0037(9)$ \\
\hline $\mathrm{C} 5$ & $0.0495(12)$ & $0.0458(12)$ & $0.0517(12)$ & $-0.0032(10)$ & $0.0082(10)$ & $-0.0044(10)$ \\
\hline C9 & $0.0482(12)$ & $0.0468(12)$ & $0.0529(12)$ & $-0.0031(10)$ & $0.0069(10)$ & $-0.0020(10)$ \\
\hline C19 & $0.0463(12)$ & $0.0451(12)$ & $0.0477(12)$ & $-0.0054(9)$ & $0.0040(9)$ & $0.0005(9)$ \\
\hline $\mathrm{C} 7$ & $0.0666(15)$ & $0.0529(14)$ & $0.0554(13)$ & $-0.0031(12)$ & $0.0159(12)$ & $0.0036(11)$ \\
\hline C13 & $0.0456(12)$ & $0.0516(13)$ & $0.0602(13)$ & $0.0111(10)$ & $0.0026(10)$ & $-0.0018(11)$ \\
\hline $\mathrm{C} 20$ & $0.0542(14)$ & $0.0591(14)$ & $0.0556(13)$ & $0.0034(11)$ & $0.0127(11)$ & $0.0019(11)$ \\
\hline $\mathrm{C} 34$ & $0.0555(13)$ & $0.0504(13)$ & $0.0429(11)$ & $-0.0032(10)$ & $0.0107(10)$ & $0.0069(10)$ \\
\hline $\mathrm{C} 33$ & $0.0518(13)$ & $0.0491(13)$ & $0.0451(11)$ & $-0.0030(10)$ & $0.0122(10)$ & $0.0058(9)$ \\
\hline C14 & $0.0584(14)$ & $0.0448(12)$ & $0.0512(12)$ & $0.0052(10)$ & $-0.0030(11)$ & $0.0024(10)$ \\
\hline $\mathrm{C} 28$ & $0.0568(14)$ & $0.0522(13)$ & $0.0481(12)$ & $-0.0015(11)$ & $0.0024(10)$ & $0.0037(10)$ \\
\hline $\mathrm{C} 21$ & $0.0619(15)$ & $0.0604(15)$ & 0.0609 (14) & $-0.0030(12)$ & $0.0092(12)$ & $0.0149(12)$ \\
\hline $\mathrm{C} 23$ & $0.0659(15)$ & $0.0548(14)$ & $0.0516(13)$ & $0.0012(12)$ & $0.0158(11)$ & $0.0046(11)$ \\
\hline $\mathrm{C} 37$ & $0.0654(15)$ & 0.0493 & $0.0453(12)$ & $-0.0020(11)$ & $0.0068(10)$ & $0.0081(10)$ \\
\hline $\mathrm{C} 35$ & 0.0597 (14) & 0.0535 (14) & $0.0547(13)$ & $-0.0080(11)$ & $0.0152(11)$ & 0.0005 (11) \\
\hline $\mathrm{C} 36$ & 0.0759 (16) & $0.0565(15)$ & $0.0606(14)$ & $-0.0040(12)$ & $0.0200(12)$ & $0.0012(11)$ \\
\hline C6 & $0.0616(15)$ & $0.0543(14)$ & $0.0615(14)$ & 0.0003 (12) & $0.0206(12)$ & $0.0030(11)$ \\
\hline C31 & $0.0526(14)$ & $0.0551(15)$ & $0.0768(16)$ & $0.0021(11)$ & $0.0180(12)$ & $-0.0005(12)$ \\
\hline $\mathrm{C} 45$ & $0.0809(18)$ & $0.0550(15)$ & $0.0648(15)$ & $0.0013(13)$ & $0.0117(13)$ & $-0.0078(13)$ \\
\hline $\mathrm{C} 4$ & $0.0469(13)$ & $0.0658(15)$ & $0.0693(15)$ & $0.0013(11)$ & $0.0150(11)$ & $-0.0054(12)$ \\
\hline $\mathrm{C} 32$ & $0.0472(13)$ & $0.0582(15)$ & $0.0630(14)$ & $-0.0043(11)$ & $0.0116(11)$ & $0.0018(11)$ \\
\hline $\mathrm{C} 22$ & $0.0651(15)$ & $0.0582(15)$ & 0.0689 (16) & $0.0053(12)$ & $0.0229(12)$ & $0.0004(12)$ \\
\hline C39 & $0.097(2)$ & $0.0438(14)$ & $0.0740(17)$ & $0.0070(14)$ & $0.0186(15)$ & $0.0115(12)$ \\
\hline $\mathrm{C} 26$ & $0.0597(16)$ & $0.081(2)$ & 0.0854 (19) & $-0.0030(14)$ & $-0.0156(14)$ & $-0.0105(16)$ \\
\hline $\mathrm{C} 42$ & $0.0752(16)$ & $0.0636(15)$ & $0.0761(15)$ & $-0.0041(12)$ & $0.0255(12)$ & $-0.0201(11)$ \\
\hline $\mathrm{C} 27$ & $0.0724(17)$ & 0.0723 (17) & $0.0637(16)$ & $0.0049(14)$ & $-0.0221(14)$ & $-0.0032(13)$ \\
\hline $\mathrm{C} 41$ & 0.0883 (19) & $0.0602(17)$ & 0.0870 (19) & $0.0006(13)$ & $0.0085(16)$ & 0.0007 (14) \\
\hline $\mathrm{C} 40$ & $0.100(2)$ & $0.0579(16)$ & $0.0699(17)$ & $-0.0029(13)$ & $0.0281(15)$ & $-0.0027(13)$ \\
\hline $\mathrm{C} 3$ & $0.0584(15)$ & $0.0798(18)$ & $0.0683(16)$ & $0.0086(13)$ & $0.0166(12)$ & $-0.0035(13)$ \\
\hline C38 & $0.0719(16)$ & $0.0569(15)$ & $0.0645(15)$ & $0.0077(13)$ & $0.0023(13)$ & $0.0149(12)$ \\
\hline $\mathrm{C} 29$ & $0.0673(16)$ & $0.0580(15)$ & $0.0661(15)$ & $-0.0050(13)$ & $-0.0032(12)$ & $-0.0074(12)$ \\
\hline $\mathrm{C} 46$ & $0.104(2)$ & $0.0522(15)$ & $0.0692(15)$ & $-0.0013(15)$ & 0.0285 & $0.0020(13)$ \\
\hline $\mathrm{C} 30$ & 0.0719 (17) & $0.0514(14)$ & $0.0755(17)$ & $-0.0023(13)$ & $0.0124(14)$ & $-0.0075(12)$ \\
\hline $\mathrm{C} 44$ & $0.085(2)$ & $0.0575(16)$ & $0.083(2)$ & $0.0022(15)$ & $0.0112(17)$ & $0.0012(14)$ \\
\hline $\mathrm{C} 43$ & $0.0782(18)$ & 0.0707 (17) & $0.0793(18)$ & $0.0019(15)$ & $0.0096(15)$ & $-0.0049(13)$ \\
\hline $\mathrm{C} 2$ & 0.0763 (19) & $0.093(2)$ & $0.0745(18)$ & $0.0062(16)$ & $0.0033(15)$ & $-0.0016(16)$ \\
\hline $\mathrm{C} 25$ & $0.080(2)$ & 0.0744 (19) & $0.087(2)$ & $-0.0147(15)$ & $-0.0064(16)$ & $-0.0077(16)$ \\
\hline $\mathrm{C} 1$ & $0.086(2)$ & $0.121(3)$ & $0.0722(18)$ & $-0.0024(19)$ & $0.0110(16)$ & $-0.0261(18)$ \\
\hline
\end{tabular}




\begin{tabular}{lllllll} 
C24 & $0.106(3)$ & $0.107(3)$ & $0.118(3)$ & $-0.028(2)$ & $0.015(2)$ & $0.000(2)$ \\
N2 & $0.0712(13)$ & $0.0541(11)$ & $0.0558(11)$ & $0.0035(10)$ & $0.0176(10)$ & $0.0101(9)$ \\
N1 & $0.0462(10)$ & $0.0529(11)$ & $0.0588(11)$ & $0.0045(9)$ & $0.0130(9)$ & $0.0007(9)$ \\
N4 & $0.0630(12)$ & $0.0545(12)$ & $0.0589(12)$ & $0.0016(10)$ & $-0.0094(10)$ & $0.0037(9)$ \\
N3 & $0.0599(12)$ & $0.0514(12)$ & $0.0753(14)$ & $0.0026(9)$ & $0.0130(11)$ & $0.0063(10)$ \\
N6 & $0.0704(14)$ & $0.0531(13)$ & $0.0771(15)$ & $-0.0013(10)$ & $0.0119(12)$ & $-0.0038(11)$ \\
N5 & $0.0544(13)$ & $0.0635(14)$ & $0.151(2)$ & $0.0097(11)$ & $0.0119(14)$ & $-0.0127(15)$ \\
\hline
\end{tabular}

Geometric parameters $\left(\AA,{ }^{\circ}\right)$

\begin{tabular}{|c|c|c|c|}
\hline $\mathrm{C} 8-\mathrm{C} 7$ & $1.403(3)$ & $\mathrm{C} 45-\mathrm{C} 46$ & $1.391(4)$ \\
\hline $\mathrm{C} 8-\mathrm{C} 9$ & $1.405(3)$ & $\mathrm{C} 45-\mathrm{H} 45$ & 0.9300 \\
\hline $\mathrm{C} 8-\mathrm{C} 17$ & $1.462(3)$ & $\mathrm{C} 4-\mathrm{N} 1$ & $1.454(3)$ \\
\hline $\mathrm{C} 10-\mathrm{C} 9$ & $1.387(3)$ & $\mathrm{C} 4-\mathrm{C} 3$ & $1.503(3)$ \\
\hline $\mathrm{C} 10-\mathrm{C} 5$ & $1.418(3)$ & $\mathrm{C} 4-\mathrm{H} 4 \mathrm{~A}$ & 0.9700 \\
\hline $\mathrm{C} 10-\mathrm{C} 11$ & $1.446(3)$ & $\mathrm{C} 4-\mathrm{H} 4 \mathrm{~B}$ & 0.9700 \\
\hline $\mathrm{C} 11-\mathrm{C} 16$ & $1.396(3)$ & $\mathrm{C} 32-\mathrm{H} 32$ & 0.9300 \\
\hline $\mathrm{C} 11-\mathrm{C} 12$ & $1.401(3)$ & $\mathrm{C} 22-\mathrm{N} 3$ & $1.334(3)$ \\
\hline $\mathrm{C} 18-\mathrm{C} 17$ & $1.323(3)$ & $\mathrm{C} 22-\mathrm{H} 22$ & 0.9300 \\
\hline $\mathrm{C} 18-\mathrm{C} 19$ & $1.474(3)$ & $\mathrm{C} 39-\mathrm{C} 38$ & $1.387(4)$ \\
\hline C18-H18 & 0.9300 & С $39-\mathrm{H} 39$ & 0.9300 \\
\hline C17-H17 & 0.9300 & $\mathrm{C} 26-\mathrm{C} 25$ & $1.517(4)$ \\
\hline $\mathrm{C} 12-\mathrm{C} 13$ & $1.389(3)$ & $\mathrm{C} 26-\mathrm{C} 27$ & $1.517(4)$ \\
\hline $\mathrm{C} 12-\mathrm{N} 1$ & $1.397(3)$ & $\mathrm{C} 26-\mathrm{H} 26 \mathrm{~A}$ & 0.9700 \\
\hline $\mathrm{C} 16-\mathrm{C} 15$ & $1.386(3)$ & $\mathrm{C} 26-\mathrm{H} 26 \mathrm{~B}$ & 0.9700 \\
\hline $\mathrm{C} 16-\mathrm{H} 16$ & 0.9300 & $\mathrm{C} 42-\mathrm{C} 43$ & $1.368(4)$ \\
\hline $\mathrm{C} 15-\mathrm{C} 14$ & $1.398(3)$ & $\mathrm{C} 42-\mathrm{C} 46$ & $1.402(4)$ \\
\hline $\mathrm{C} 15-\mathrm{N} 2$ & $1.403(3)$ & $\mathrm{C} 42-\mathrm{C} 41$ & $1.482(4)$ \\
\hline $\mathrm{C} 5-\mathrm{N} 1$ & $1.377(3)$ & $\mathrm{C} 27-\mathrm{N} 4$ & $1.456(3)$ \\
\hline $\mathrm{C} 5-\mathrm{C} 6$ & $1.396(3)$ & $\mathrm{C} 27-\mathrm{H} 27 \mathrm{~A}$ & 0.9700 \\
\hline C9-H9 & 0.9300 & $\mathrm{C} 27-\mathrm{H} 27 \mathrm{~B}$ & 0.9700 \\
\hline $\mathrm{C} 19-\mathrm{C} 23$ & $1.382(3)$ & $\mathrm{C} 41-\mathrm{C} 40$ & 1.269 (4) \\
\hline $\mathrm{C} 19-\mathrm{C} 20$ & $1.388(3)$ & $\mathrm{C} 41-\mathrm{H} 41$ & 0.9300 \\
\hline $\mathrm{C} 7-\mathrm{C} 6$ & $1.363(3)$ & $\mathrm{C} 40-\mathrm{H} 40$ & 0.9300 \\
\hline $\mathrm{C} 7-\mathrm{H} 7$ & 0.9300 & $\mathrm{C} 3-\mathrm{C} 2$ & $1.480(4)$ \\
\hline $\mathrm{C} 13-\mathrm{C} 14$ & $1.380(3)$ & $\mathrm{C} 3-\mathrm{H} 3 \mathrm{~A}$ & 0.9700 \\
\hline $\mathrm{C} 13-\mathrm{H} 13$ & 0.9300 & $\mathrm{C} 3-\mathrm{H} 3 \mathrm{~B}$ & 0.9700 \\
\hline $\mathrm{C} 20-\mathrm{C} 21$ & $1.372(3)$ & $\mathrm{C} 38-\mathrm{H} 38$ & 0.9300 \\
\hline $\mathrm{C} 20-\mathrm{H} 20$ & 0.9300 & $\mathrm{C} 29-\mathrm{C} 30$ & $1.362(4)$ \\
\hline $\mathrm{C} 34-\mathrm{C} 35$ & $1.386(3)$ & $\mathrm{C} 29-\mathrm{H} 29$ & 0.9300 \\
\hline $\mathrm{C} 34-\mathrm{C} 37$ & $1.410(3)$ & $\mathrm{C} 46-\mathrm{H} 46$ & 0.9300 \\
\hline $\mathrm{C} 34-\mathrm{C} 33$ & $1.439(3)$ & $\mathrm{C} 30-\mathrm{H} 30$ & 0.9300 \\
\hline $\mathrm{C} 33-\mathrm{C} 32$ & $1.396(3)$ & $\mathrm{C} 44-\mathrm{N} 6$ & $1.321(4)$ \\
\hline $\mathrm{C} 33-\mathrm{C} 28$ & $1.402(3)$ & $\mathrm{C} 44-\mathrm{C} 43$ & $1.350(4)$ \\
\hline C14-H14 & 0.9300 & $\mathrm{C} 44-\mathrm{H} 44$ & 0.9300 \\
\hline $\mathrm{C} 28-\mathrm{C} 29$ & $1.388(3)$ & $\mathrm{C} 43-\mathrm{H} 43$ & 0.9300 \\
\hline $\mathrm{C} 28-\mathrm{N} 4$ & $1.393(3)$ & $\mathrm{C} 2-\mathrm{C} 1$ & $1.534(4)$ \\
\hline $\mathrm{C} 21-\mathrm{N} 3$ & $1.342(3)$ & $\mathrm{C} 2-\mathrm{H} 2 \mathrm{~A}$ & 0.9700 \\
\hline
\end{tabular}




\begin{tabular}{|c|c|c|c|}
\hline $\mathrm{C} 21-\mathrm{H} 21$ & 0.9300 & $\mathrm{C} 2-\mathrm{H} 2 \mathrm{~B}$ & 0.9700 \\
\hline $\mathrm{C} 23-\mathrm{C} 22$ & $1.369(3)$ & $\mathrm{C} 25-\mathrm{C} 24$ & $1.514(5)$ \\
\hline $\mathrm{C} 23-\mathrm{H} 23$ & 0.9300 & $\mathrm{C} 25-\mathrm{H} 25 \mathrm{~A}$ & 0.9700 \\
\hline $\mathrm{C} 37-\mathrm{N} 4$ & $1.378(3)$ & $\mathrm{C} 25-\mathrm{H} 25 \mathrm{~B}$ & 0.9700 \\
\hline $\mathrm{C} 37-\mathrm{C} 38$ & $1.391(3)$ & $\mathrm{C} 1-\mathrm{H} 1 \mathrm{~A}$ & 0.9600 \\
\hline $\mathrm{C} 35-\mathrm{C} 36$ & $1.374(3)$ & $\mathrm{C} 1-\mathrm{H} 1 \mathrm{~B}$ & 0.9600 \\
\hline $\mathrm{C} 35-\mathrm{H} 35$ & 0.9300 & $\mathrm{C} 1-\mathrm{H} 1 \mathrm{C}$ & 0.9600 \\
\hline $\mathrm{C} 36-\mathrm{C} 39$ & $1.407(4)$ & $\mathrm{C} 24-\mathrm{H} 24 \mathrm{~A}$ & 0.9600 \\
\hline $\mathrm{C} 36-\mathrm{C} 40$ & $1.503(4)$ & $\mathrm{C} 24-\mathrm{H} 24 \mathrm{~B}$ & 0.9600 \\
\hline $\mathrm{C} 6-\mathrm{H} 6$ & 0.9300 & $\mathrm{C} 24-\mathrm{H} 24 \mathrm{C}$ & 0.9600 \\
\hline $\mathrm{C} 31-\mathrm{C} 32$ & $1.381(3)$ & $\mathrm{N} 2-\mathrm{H} 2 \mathrm{C}$ & 0.8600 \\
\hline $\mathrm{C} 31-\mathrm{N} 5$ & $1.395(3)$ & $\mathrm{N} 2-\mathrm{H} 2 \mathrm{D}$ & 0.8600 \\
\hline $\mathrm{C} 31-\mathrm{C} 30$ & $1.402(4)$ & $\mathrm{N} 5-\mathrm{H} 5 \mathrm{~A}$ & 0.8600 \\
\hline $\mathrm{C} 45-\mathrm{N} 6$ & $1.327(3)$ & $\mathrm{N} 5-\mathrm{H} 5 \mathrm{~B}$ & 0.8600 \\
\hline $\mathrm{C} 7-\mathrm{C} 8-\mathrm{C} 9$ & $118.4(2)$ & $\mathrm{N} 3-\mathrm{C} 22-\mathrm{H} 22$ & 117.9 \\
\hline $\mathrm{C} 7-\mathrm{C} 8-\mathrm{C} 17$ & $118.7(2)$ & $\mathrm{C} 23-\mathrm{C} 22-\mathrm{H} 22$ & 117.9 \\
\hline $\mathrm{C} 9-\mathrm{C} 8-\mathrm{C} 17$ & $122.8(2)$ & $\mathrm{C} 38-\mathrm{C} 39-\mathrm{C} 36$ & $123.0(2)$ \\
\hline $\mathrm{C} 9-\mathrm{C} 10-\mathrm{C} 5$ & $119.92(19)$ & $\mathrm{C} 38-\mathrm{C} 39-\mathrm{H} 39$ & 118.5 \\
\hline $\mathrm{C} 9-\mathrm{C} 10-\mathrm{C} 11$ & $133.71(19)$ & $\mathrm{C} 36-\mathrm{C} 39-\mathrm{H} 39$ & 118.5 \\
\hline $\mathrm{C} 5-\mathrm{C} 10-\mathrm{C} 11$ & $106.36(18)$ & $\mathrm{C} 25-\mathrm{C} 26-\mathrm{C} 27$ & $114.7(2)$ \\
\hline $\mathrm{C} 16-\mathrm{C} 11-\mathrm{C} 12$ & $119.76(19)$ & $\mathrm{C} 25-\mathrm{C} 26-\mathrm{H} 26 \mathrm{~A}$ & 108.6 \\
\hline $\mathrm{C} 16-\mathrm{C} 11-\mathrm{C} 10$ & $133.52(19)$ & $\mathrm{C} 27-\mathrm{C} 26-\mathrm{H} 26 \mathrm{~A}$ & 108.6 \\
\hline $\mathrm{C} 12-\mathrm{C} 11-\mathrm{C} 10$ & $106.71(18)$ & $\mathrm{C} 25-\mathrm{C} 26-\mathrm{H} 26 \mathrm{~B}$ & 108.6 \\
\hline $\mathrm{C} 17-\mathrm{C} 18-\mathrm{C} 19$ & $126.2(2)$ & $\mathrm{C} 27-\mathrm{C} 26-\mathrm{H} 26 \mathrm{~B}$ & 108.6 \\
\hline $\mathrm{C} 17-\mathrm{C} 18-\mathrm{H} 18$ & 116.9 & $\mathrm{H} 26 \mathrm{~A}-\mathrm{C} 26-\mathrm{H} 26 \mathrm{~B}$ & 107.6 \\
\hline $\mathrm{C} 19-\mathrm{C} 18-\mathrm{H} 18$ & 116.9 & $\mathrm{C} 43-\mathrm{C} 42-\mathrm{C} 46$ & $116.3(3)$ \\
\hline $\mathrm{C} 18-\mathrm{C} 17-\mathrm{C} 8$ & $127.9(2)$ & $\mathrm{C} 43-\mathrm{C} 42-\mathrm{C} 41$ & $118.7(3)$ \\
\hline $\mathrm{C} 18-\mathrm{C} 17-\mathrm{H} 17$ & 116.0 & $\mathrm{C} 46-\mathrm{C} 42-\mathrm{C} 41$ & $125.1(3)$ \\
\hline $\mathrm{C} 8-\mathrm{C} 17-\mathrm{H} 17$ & 116.0 & $\mathrm{~N} 4-\mathrm{C} 27-\mathrm{C} 26$ & $113.6(2)$ \\
\hline $\mathrm{C} 13-\mathrm{C} 12-\mathrm{N} 1$ & $129.8(2)$ & $\mathrm{N} 4-\mathrm{C} 27-\mathrm{H} 27 \mathrm{~A}$ & 108.8 \\
\hline $\mathrm{C} 13-\mathrm{C} 12-\mathrm{C} 11$ & $120.9(2)$ & $\mathrm{C} 26-\mathrm{C} 27-\mathrm{H} 27 \mathrm{~A}$ & 108.8 \\
\hline $\mathrm{N} 1-\mathrm{C} 12-\mathrm{C} 11$ & $109.33(18)$ & $\mathrm{N} 4-\mathrm{C} 27-\mathrm{H} 27 \mathrm{~B}$ & 108.8 \\
\hline $\mathrm{C} 15-\mathrm{C} 16-\mathrm{C} 11$ & $119.7(2)$ & $\mathrm{C} 26-\mathrm{C} 27-\mathrm{H} 27 \mathrm{~B}$ & 108.8 \\
\hline $\mathrm{C} 15-\mathrm{C} 16-\mathrm{H} 16$ & 120.1 & $\mathrm{H} 27 \mathrm{~A}-\mathrm{C} 27-\mathrm{H} 27 \mathrm{~B}$ & 107.7 \\
\hline $\mathrm{C} 11-\mathrm{C} 16-\mathrm{H} 16$ & 120.1 & $\mathrm{C} 40-\mathrm{C} 41-\mathrm{C} 42$ & $124.1(3)$ \\
\hline $\mathrm{C} 16-\mathrm{C} 15-\mathrm{C} 14$ & $119.4(2)$ & $\mathrm{C} 40-\mathrm{C} 41-\mathrm{H} 41$ & 117.9 \\
\hline $\mathrm{C} 16-\mathrm{C} 15-\mathrm{N} 2$ & $120.6(2)$ & $\mathrm{C} 42-\mathrm{C} 41-\mathrm{H} 41$ & 117.9 \\
\hline $\mathrm{C} 14-\mathrm{C} 15-\mathrm{N} 2$ & $120.1(2)$ & $\mathrm{C} 41-\mathrm{C} 40-\mathrm{C} 36$ & $127.2(3)$ \\
\hline $\mathrm{N} 1-\mathrm{C} 5-\mathrm{C} 6$ & $130.1(2)$ & $\mathrm{C} 41-\mathrm{C} 40-\mathrm{H} 40$ & 116.4 \\
\hline $\mathrm{N} 1-\mathrm{C} 5-\mathrm{C} 10$ & $109.32(18)$ & $\mathrm{C} 36-\mathrm{C} 40-\mathrm{H} 40$ & 116.4 \\
\hline $\mathrm{C} 6-\mathrm{C} 5-\mathrm{C} 10$ & $120.6(2)$ & $\mathrm{C} 2-\mathrm{C} 3-\mathrm{C} 4$ & $114.3(2)$ \\
\hline $\mathrm{C} 10-\mathrm{C} 9-\mathrm{C} 8$ & $119.7(2)$ & $\mathrm{C} 2-\mathrm{C} 3-\mathrm{H} 3 \mathrm{~A}$ & 108.7 \\
\hline $\mathrm{C} 10-\mathrm{C} 9-\mathrm{H} 9$ & 120.2 & $\mathrm{C} 4-\mathrm{C} 3-\mathrm{H} 3 \mathrm{~A}$ & 108.7 \\
\hline $\mathrm{C} 8-\mathrm{C} 9-\mathrm{H} 9$ & 120.2 & $\mathrm{C} 2-\mathrm{C} 3-\mathrm{H} 3 \mathrm{~B}$ & 108.7 \\
\hline $\mathrm{C} 23-\mathrm{C} 19-\mathrm{C} 20$ & $115.3(2)$ & $\mathrm{C} 4-\mathrm{C} 3-\mathrm{H} 3 \mathrm{~B}$ & 108.7 \\
\hline $\mathrm{C} 23-\mathrm{C} 19-\mathrm{C} 18$ & $120.56(19)$ & $\mathrm{H} 3 \mathrm{~A}-\mathrm{C} 3-\mathrm{H} 3 \mathrm{~B}$ & 107.6 \\
\hline $\mathrm{C} 20-\mathrm{C} 19-\mathrm{C} 18$ & $124.1(2)$ & $\mathrm{C} 39-\mathrm{C} 38-\mathrm{C} 37$ & $117.3(2)$ \\
\hline
\end{tabular}




\begin{tabular}{|c|c|}
\hline $\mathrm{C} 6-\mathrm{C} 7-\mathrm{C} 8$ & $123.2(2)$ \\
\hline $\mathrm{C} 6-\mathrm{C} 7-\mathrm{H} 7$ & 118.4 \\
\hline $\mathrm{C} 8-\mathrm{C} 7-\mathrm{H} 7$ & 118.4 \\
\hline $\mathrm{C} 14-\mathrm{C} 13-\mathrm{C} 12$ & $118.3(2)$ \\
\hline $\mathrm{C} 14-\mathrm{C} 13-\mathrm{H} 13$ & 120.8 \\
\hline $\mathrm{C} 12-\mathrm{C} 13-\mathrm{H} 13$ & 120.8 \\
\hline $\mathrm{C} 21-\mathrm{C} 20-\mathrm{C} 19$ & $120.1(2)$ \\
\hline $\mathrm{C} 21-\mathrm{C} 20-\mathrm{H} 20$ & 119.9 \\
\hline $\mathrm{C} 19-\mathrm{C} 20-\mathrm{H} 20$ & 119.9 \\
\hline $\mathrm{C} 35-\mathrm{C} 34-\mathrm{C} 37$ & $120.0(2)$ \\
\hline $\mathrm{C} 35-\mathrm{C} 34-\mathrm{C} 33$ & $133.2(2)$ \\
\hline $\mathrm{C} 37-\mathrm{C} 34-\mathrm{C} 33$ & $106.7(2)$ \\
\hline $\mathrm{C} 32-\mathrm{C} 33-\mathrm{C} 28$ & $120.0(2)$ \\
\hline $\mathrm{C} 32-\mathrm{C} 33-\mathrm{C} 34$ & $133.5(2)$ \\
\hline $\mathrm{C} 28-\mathrm{C} 33-\mathrm{C} 34$ & $106.4(2)$ \\
\hline $\mathrm{C} 13-\mathrm{C} 14-\mathrm{C} 15$ & $121.9(2)$ \\
\hline $\mathrm{C} 13-\mathrm{C} 14-\mathrm{H} 14$ & 119.0 \\
\hline $\mathrm{C} 15-\mathrm{C} 14-\mathrm{H} 14$ & 119.0 \\
\hline $\mathrm{C} 29-\mathrm{C} 28-\mathrm{N} 4$ & $130.1(2)$ \\
\hline $\mathrm{C} 29-\mathrm{C} 28-\mathrm{C} 33$ & $120.4(2)$ \\
\hline $\mathrm{N} 4-\mathrm{C} 28-\mathrm{C} 33$ & $109.5(2)$ \\
\hline $\mathrm{N} 3-\mathrm{C} 21-\mathrm{C} 20$ & $124.6(2)$ \\
\hline $\mathrm{N} 3-\mathrm{C} 21-\mathrm{H} 21$ & 117.7 \\
\hline $\mathrm{C} 20-\mathrm{C} 21-\mathrm{H} 21$ & 117.7 \\
\hline $\mathrm{C} 22-\mathrm{C} 23-\mathrm{C} 19$ & $121.0(2)$ \\
\hline $\mathrm{C} 22-\mathrm{C} 23-\mathrm{H} 23$ & 119.5 \\
\hline $\mathrm{C} 19-\mathrm{C} 23-\mathrm{H} 23$ & 119.5 \\
\hline $\mathrm{N} 4-\mathrm{C} 37-\mathrm{C} 38$ & $129.9(2)$ \\
\hline $\mathrm{N} 4-\mathrm{C} 37-\mathrm{C} 34$ & $109.4(2)$ \\
\hline $\mathrm{C} 38-\mathrm{C} 37-\mathrm{C} 34$ & $120.7(2)$ \\
\hline $\mathrm{C} 36-\mathrm{C} 35-\mathrm{C} 34$ & $120.6(2)$ \\
\hline $\mathrm{C} 36-\mathrm{C} 35-\mathrm{H} 35$ & 119.7 \\
\hline C34-C35-H35 & 119.7 \\
\hline $\mathrm{C} 35-\mathrm{C} 36-\mathrm{C} 39$ & $118.2(2)$ \\
\hline $\mathrm{C} 35-\mathrm{C} 36-\mathrm{C} 40$ & $117.4(2)$ \\
\hline $\mathrm{C} 39-\mathrm{C} 36-\mathrm{C} 40$ & $124.3(2)$ \\
\hline $\mathrm{C} 7-\mathrm{C} 6-\mathrm{C} 5$ & $118.1(2)$ \\
\hline $\mathrm{C} 7-\mathrm{C} 6-\mathrm{H} 6$ & 120.9 \\
\hline $\mathrm{C} 5-\mathrm{C} 6-\mathrm{H} 6$ & 120.9 \\
\hline $\mathrm{C} 32-\mathrm{C} 31-\mathrm{N} 5$ & $121.5(2)$ \\
\hline $\mathrm{C} 32-\mathrm{C} 31-\mathrm{C} 30$ & $119.0(2)$ \\
\hline $\mathrm{N} 5-\mathrm{C} 31-\mathrm{C} 30$ & $119.5(2)$ \\
\hline $\mathrm{N} 6-\mathrm{C} 45-\mathrm{C} 46$ & $122.9(3)$ \\
\hline $\mathrm{N} 6-\mathrm{C} 45-\mathrm{H} 45$ & 118.5 \\
\hline $\mathrm{C} 46-\mathrm{C} 45-\mathrm{H} 45$ & 118.5 \\
\hline $\mathrm{N} 1-\mathrm{C} 4-\mathrm{C} 3$ & $114.8(2)$ \\
\hline $\mathrm{N} 1-\mathrm{C} 4-\mathrm{H} 4 \mathrm{~A}$ & 108.6 \\
\hline 4 & 108.6 \\
\hline
\end{tabular}

$\begin{array}{ll}\mathrm{C} 39-\mathrm{C} 38-\mathrm{H} 38 & 121.3 \\ \mathrm{C} 37-\mathrm{C} 38-\mathrm{H} 38 & 121.3 \\ \mathrm{C} 30-\mathrm{C} 29-\mathrm{C} 28 & 118.7(2) \\ \mathrm{C} 30-\mathrm{C} 29-\mathrm{H} 29 & 120.7 \\ \mathrm{C} 28-\mathrm{C} 29-\mathrm{H} 29 & 120.7 \\ \mathrm{C} 45-\mathrm{C} 46-\mathrm{C} 42 & 119.4(3) \\ \mathrm{C} 45-\mathrm{C} 46-\mathrm{H} 46 & 120.3 \\ \mathrm{C} 42-\mathrm{C} 46-\mathrm{H} 46 & 120.3 \\ \mathrm{C} 29-\mathrm{C} 30-\mathrm{C} 31 & 122.4(2) \\ \mathrm{C} 29-\mathrm{C} 30-\mathrm{H} 30 & 118.8 \\ \mathrm{C} 31-\mathrm{C} 30-\mathrm{H} 30 & 118.8 \\ \mathrm{~N} 6-\mathrm{C} 44-\mathrm{C} 43 & 125.6(3) \\ \mathrm{N} 6-\mathrm{C} 44-\mathrm{H} 44 & 117.2\end{array}$

117.2

117.2

119.8 (3)

120.1

120.1

112.9 (3)

109.0

109.0

109.0

109.0

107.8

114.2 (3)

108.7

108.7

108.7

108.7

107.6

109.5

109.5

109.5

109.5

109.5

109.5

109.5

109.5

109.5

109.5

109.5

109.5

120.0

120.0

120.0

108.27 (17)

127.33 (19)

124.32 (19)

107.96 (19) 


\begin{tabular}{|c|c|}
\hline $\mathrm{N} 1-\mathrm{C} 4-\mathrm{H} 4 \mathrm{~B}$ & 108.6 \\
\hline $\mathrm{C} 3-\mathrm{C} 4-\mathrm{H} 4 \mathrm{~B}$ & 108.6 \\
\hline $\mathrm{H} 4 \mathrm{~A}-\mathrm{C} 4-\mathrm{H} 4 \mathrm{~B}$ & 107.5 \\
\hline $\mathrm{C} 31-\mathrm{C} 32-\mathrm{C} 33$ & $119.6(2)$ \\
\hline $\mathrm{C} 31-\mathrm{C} 32-\mathrm{H} 32$ & 120.2 \\
\hline $\mathrm{C} 33-\mathrm{C} 32-\mathrm{H} 32$ & 120.2 \\
\hline $\mathrm{N} 3-\mathrm{C} 22-\mathrm{C} 23$ & $124.2(2)$ \\
\hline $\mathrm{C} 9-\mathrm{C} 10-\mathrm{C} 11-\mathrm{C} 16$ & $-3.2(4)$ \\
\hline $\mathrm{C} 5-\mathrm{C} 10-\mathrm{C} 11-\mathrm{C} 16$ & $178.0(2)$ \\
\hline $\mathrm{C} 9-\mathrm{C} 10-\mathrm{C} 11-\mathrm{C} 12$ & $178.4(2)$ \\
\hline $\mathrm{C} 5-\mathrm{C} 10-\mathrm{C} 11-\mathrm{C} 12$ & $-0.4(2)$ \\
\hline $\mathrm{C} 19-\mathrm{C} 18-\mathrm{C} 17-\mathrm{C} 8$ & $-178.2(2)$ \\
\hline $\mathrm{C} 7-\mathrm{C} 8-\mathrm{C} 17-\mathrm{C} 18$ & $163.1(2)$ \\
\hline $\mathrm{C} 9-\mathrm{C} 8-\mathrm{C} 17-\mathrm{C} 18$ & $-14.6(4)$ \\
\hline $\mathrm{C} 16-\mathrm{C} 11-\mathrm{C} 12-\mathrm{C} 13$ & $0.4(3)$ \\
\hline $\mathrm{C} 10-\mathrm{C} 11-\mathrm{C} 12-\mathrm{C} 13$ & $179.04(19)$ \\
\hline $\mathrm{C} 16-\mathrm{C} 11-\mathrm{C} 12-\mathrm{N} 1$ & $-178.96(18)$ \\
\hline $\mathrm{C} 10-\mathrm{C} 11-\mathrm{C} 12-\mathrm{N} 1$ & $-0.3(2)$ \\
\hline $\mathrm{C} 12-\mathrm{C} 11-\mathrm{C} 16-\mathrm{C} 15$ & $-0.2(3)$ \\
\hline $\mathrm{C} 10-\mathrm{C} 11-\mathrm{C} 16-\mathrm{C} 15$ & $-178.4(2)$ \\
\hline $\mathrm{C} 11-\mathrm{C} 16-\mathrm{C} 15-\mathrm{C} 14$ & $-0.3(3)$ \\
\hline $\mathrm{C} 11-\mathrm{C} 16-\mathrm{C} 15-\mathrm{N} 2$ & $-178.79(19)$ \\
\hline $\mathrm{C} 9-\mathrm{C} 10-\mathrm{C} 5-\mathrm{N} 1$ & $-177.99(18)$ \\
\hline $\mathrm{C} 11-\mathrm{C} 10-\mathrm{C} 5-\mathrm{N} 1$ & $1.0(2)$ \\
\hline $\mathrm{C} 9-\mathrm{C} 10-\mathrm{C} 5-\mathrm{C} 6$ & $2.5(3)$ \\
\hline $\mathrm{C} 11-\mathrm{C} 10-\mathrm{C} 5-\mathrm{C} 6$ & $-178.5(2)$ \\
\hline $\mathrm{C} 5-\mathrm{C} 10-\mathrm{C} 9-\mathrm{C} 8$ & $-1.2(3)$ \\
\hline $\mathrm{C} 11-\mathrm{C} 10-\mathrm{C} 9-\mathrm{C} 8$ & $-179.9(2)$ \\
\hline $\mathrm{C} 7-\mathrm{C} 8-\mathrm{C} 9-\mathrm{C} 10$ & $-0.8(3)$ \\
\hline $\mathrm{C} 17-\mathrm{C} 8-\mathrm{C} 9-\mathrm{C} 10$ & $176.8(2)$ \\
\hline $\mathrm{C} 17-\mathrm{C} 18-\mathrm{C} 19-\mathrm{C} 23$ & $176.3(2)$ \\
\hline $\mathrm{C} 17-\mathrm{C} 18-\mathrm{C} 19-\mathrm{C} 20$ & $-3.5(4)$ \\
\hline $\mathrm{C} 9-\mathrm{C} 8-\mathrm{C} 7-\mathrm{C} 6$ & $1.7(3)$ \\
\hline $\mathrm{C} 17-\mathrm{C} 8-\mathrm{C} 7-\mathrm{C} 6$ & $-176.0(2)$ \\
\hline $\mathrm{N} 1-\mathrm{C} 12-\mathrm{C} 13-\mathrm{C} 14$ & $179.1(2)$ \\
\hline $\mathrm{C} 11-\mathrm{C} 12-\mathrm{C} 13-\mathrm{C} 14$ & $-0.1(3)$ \\
\hline $\mathrm{C} 23-\mathrm{C} 19-\mathrm{C} 20-\mathrm{C} 21$ & $0.9(3)$ \\
\hline $\mathrm{C} 18-\mathrm{C} 19-\mathrm{C} 20-\mathrm{C} 21$ & $-179.3(2)$ \\
\hline $\mathrm{C} 35-\mathrm{C} 34-\mathrm{C} 33-\mathrm{C} 32$ & $-1.8(4)$ \\
\hline $\mathrm{C} 37-\mathrm{C} 34-\mathrm{C} 33-\mathrm{C} 32$ & $-177.7(2)$ \\
\hline $\mathrm{C} 35-\mathrm{C} 34-\mathrm{C} 33-\mathrm{C} 28$ & $175.2(2)$ \\
\hline $\mathrm{C} 37-\mathrm{C} 34-\mathrm{C} 33-\mathrm{C} 28$ & $-0.7(2)$ \\
\hline $\mathrm{C} 12-\mathrm{C} 13-\mathrm{C} 14-\mathrm{C} 15$ & $-0.3(3)$ \\
\hline $\mathrm{C} 16-\mathrm{C} 15-\mathrm{C} 14-\mathrm{C} 13$ & $0.5(3)$ \\
\hline $\mathrm{N} 2-\mathrm{C} 15-\mathrm{C} 14-\mathrm{C} 13$ & $179.1(2)$ \\
\hline $\mathrm{C} 32-\mathrm{C} 33-\mathrm{C} 28-\mathrm{C} 29$ & $0.4(3)$ \\
\hline $28-$ & \\
\hline
\end{tabular}

\begin{tabular}{|c|c|}
\hline $\mathrm{C} 37-\mathrm{N} 4-\mathrm{C} 27$ & $126.1(2)$ \\
\hline $\mathrm{C} 28-\mathrm{N} 4-\mathrm{C} 27$ & $125.9(2)$ \\
\hline $\mathrm{C} 22-\mathrm{N} 3-\mathrm{C} 21$ & $114.8(2)$ \\
\hline $\mathrm{C} 44-\mathrm{N} 6-\mathrm{C} 45$ & $115.9(2)$ \\
\hline $\mathrm{C} 31-\mathrm{N} 5-\mathrm{H} 5 \mathrm{~A}$ & 120.0 \\
\hline $\mathrm{C} 31-\mathrm{N} 5-\mathrm{H} 5 \mathrm{~B}$ & 120.0 \\
\hline $\mathrm{H} 5 \mathrm{~A}-\mathrm{N} 5-\mathrm{H} 5 \mathrm{~B}$ & 120.0 \\
\hline $\mathrm{C} 10-\mathrm{C} 5-\mathrm{C} 6-\mathrm{C} 7$ & $-1.7(3)$ \\
\hline $\mathrm{N} 5-\mathrm{C} 31-\mathrm{C} 32-\mathrm{C} 33$ & $179.7(2)$ \\
\hline $\mathrm{C} 30-\mathrm{C} 31-\mathrm{C} 32-\mathrm{C} 33$ & $-1.6(3)$ \\
\hline $\mathrm{C} 28-\mathrm{C} 33-\mathrm{C} 32-\mathrm{C} 31$ & $0.5(3)$ \\
\hline $\mathrm{C} 34-\mathrm{C} 33-\mathrm{C} 32-\mathrm{C} 31$ & $177.2(2)$ \\
\hline $\mathrm{C} 19-\mathrm{C} 23-\mathrm{C} 22-\mathrm{N} 3$ & $-0.4(4)$ \\
\hline $\mathrm{C} 35-\mathrm{C} 36-\mathrm{C} 39-\mathrm{C} 38$ & $2.3(4)$ \\
\hline $\mathrm{C} 40-\mathrm{C} 36-\mathrm{C} 39-\mathrm{C} 38$ & $-173.6(2)$ \\
\hline $\mathrm{C} 25-\mathrm{C} 26-\mathrm{C} 27-\mathrm{N} 4$ & $61.0(3)$ \\
\hline $\mathrm{C} 43-\mathrm{C} 42-\mathrm{C} 41-\mathrm{C} 40$ & $-160.0(3)$ \\
\hline $\mathrm{C} 46-\mathrm{C} 42-\mathrm{C} 41-\mathrm{C} 40$ & $19.3(4)$ \\
\hline $\mathrm{C} 42-\mathrm{C} 41-\mathrm{C} 40-\mathrm{C} 36$ & $174.1(2)$ \\
\hline $\mathrm{C} 35-\mathrm{C} 36-\mathrm{C} 40-\mathrm{C} 41$ & $-151.0(3)$ \\
\hline $\mathrm{C} 39-\mathrm{C} 36-\mathrm{C} 40-\mathrm{C} 41$ & $24.9(4)$ \\
\hline $\mathrm{N} 1-\mathrm{C} 4-\mathrm{C} 3-\mathrm{C} 2$ & $-63.4(3)$ \\
\hline $\mathrm{C} 36-\mathrm{C} 39-\mathrm{C} 38-\mathrm{C} 37$ & $1.4(4)$ \\
\hline $\mathrm{N} 4-\mathrm{C} 37-\mathrm{C} 38-\mathrm{C} 39$ & $174.3(2)$ \\
\hline $\mathrm{C} 34-\mathrm{C} 37-\mathrm{C} 38-\mathrm{C} 39$ & $-3.2(3)$ \\
\hline $\mathrm{N} 4-\mathrm{C} 28-\mathrm{C} 29-\mathrm{C} 30$ & $-178.0(2)$ \\
\hline $\mathrm{C} 33-\mathrm{C} 28-\mathrm{C} 29-\mathrm{C} 30$ & $-0.2(4)$ \\
\hline $\mathrm{N} 6-\mathrm{C} 45-\mathrm{C} 46-\mathrm{C} 42$ & $0.8(4)$ \\
\hline $\mathrm{C} 43-\mathrm{C} 42-\mathrm{C} 46-\mathrm{C} 45$ & $-1.4(4)$ \\
\hline $\mathrm{C} 41-\mathrm{C} 42-\mathrm{C} 46-\mathrm{C} 45$ & $179.3(2)$ \\
\hline $\mathrm{C} 28-\mathrm{C} 29-\mathrm{C} 30-\mathrm{C} 31$ & $-0.9(4)$ \\
\hline $\mathrm{C} 32-\mathrm{C} 31-\mathrm{C} 30-\mathrm{C} 29$ & $1.8(4)$ \\
\hline $\mathrm{N} 5-\mathrm{C} 31-\mathrm{C} 30-\mathrm{C} 29$ & $-179.4(3)$ \\
\hline $\mathrm{N} 6-\mathrm{C} 44-\mathrm{C} 43-\mathrm{C} 42$ & $0.1(4)$ \\
\hline $\mathrm{C} 46-\mathrm{C} 42-\mathrm{C} 43-\mathrm{C} 44$ & $0.9(4)$ \\
\hline $\mathrm{C} 41-\mathrm{C} 42-\mathrm{C} 43-\mathrm{C} 44$ & $-179.7(3)$ \\
\hline $\mathrm{C} 4-\mathrm{C} 3-\mathrm{C} 2-\mathrm{C} 1$ & $-171.7(2)$ \\
\hline $\mathrm{C} 27-\mathrm{C} 26-\mathrm{C} 25-\mathrm{C} 24$ & $173.8(3)$ \\
\hline $\mathrm{C} 6-\mathrm{C} 5-\mathrm{N} 1-\mathrm{C} 12$ & $178.2(2)$ \\
\hline $\mathrm{C} 10-\mathrm{C} 5-\mathrm{N} 1-\mathrm{C} 12$ & $-1.2(2)$ \\
\hline $\mathrm{C} 6-\mathrm{C} 5-\mathrm{N} 1-\mathrm{C} 4$ & $-5.0(4)$ \\
\hline $\mathrm{C} 10-\mathrm{C} 5-\mathrm{N} 1-\mathrm{C} 4$ & $175.5(2)$ \\
\hline $\mathrm{C} 13-\mathrm{C} 12-\mathrm{N} 1-\mathrm{C} 5$ & $-178.3(2)$ \\
\hline $\mathrm{C} 11-\mathrm{C} 12-\mathrm{N} 1-\mathrm{C} 5$ & $0.9(2)$ \\
\hline $\mathrm{C} 13-\mathrm{C} 12-\mathrm{N} 1-\mathrm{C} 4$ & $4.8(4)$ \\
\hline $\mathrm{C} 11-\mathrm{C} 12-\mathrm{N} 1-\mathrm{C} 4$ & $-175.9(2)$ \\
\hline $\mathrm{C} 3-\mathrm{C} 4-\mathrm{N} 1-\mathrm{C} 5$ & $103.0(3)$ \\
\hline
\end{tabular}




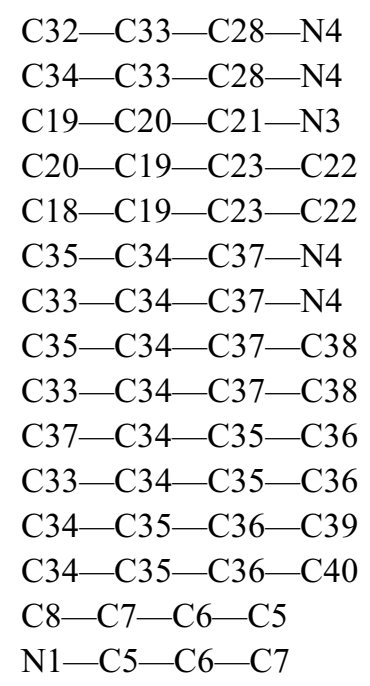

$178.61(19)$
$1.2(2)$
$0.3(4)$
$-0.9(3)$
$179.3(2)$
$-176.53(19)$
$0.0(2)$
$1.4(3)$
$177.9(2)$
$2.4(3)$
$-173.0(2)$
$-4.2(3)$
$172.0(2)$
$-0.5(4)$
$179.0(2)$

$\mathrm{C} 3-\mathrm{C} 4-\mathrm{N} 1-\mathrm{C} 12$
$\mathrm{C} 38-\mathrm{C} 37-\mathrm{N} 4-\mathrm{C} 28$
$\mathrm{C} 34-\mathrm{C} 37-\mathrm{N} 4-\mathrm{C} 28$
$\mathrm{C} 38-\mathrm{C} 37-\mathrm{N} 4-\mathrm{C} 27$
$\mathrm{C} 34-\mathrm{C} 37-\mathrm{N} 4-\mathrm{C} 27$
$\mathrm{C} 29-\mathrm{C} 28-\mathrm{N} 4-\mathrm{C} 37$
$\mathrm{C} 33-\mathrm{C} 28-\mathrm{N} 4-\mathrm{C} 37$
$\mathrm{C} 29-\mathrm{C} 28-\mathrm{N} 4-\mathrm{C} 27$
$\mathrm{C} 33-\mathrm{C} 28-\mathrm{N} 4-\mathrm{C} 27$
$\mathrm{C} 26-\mathrm{C} 27-\mathrm{N} 4-\mathrm{C} 37$
$\mathrm{C} 26-\mathrm{C} 27-\mathrm{N} 4-\mathrm{C} 28$
$\mathrm{C} 23-\mathrm{C} 22-\mathrm{N} 3-\mathrm{C} 21$
$\mathrm{C} 20-\mathrm{C} 21-\mathrm{N} 3-\mathrm{C} 22$
$\mathrm{C} 43-\mathrm{C} 44-\mathrm{N} 6-\mathrm{C} 45$
$\mathrm{C} 46-\mathrm{C} 45-\mathrm{N} 6-\mathrm{C} 44$

$-80.8(3)$

$-176.9(2)$

$0.7(2)$

$4.4(4)$

$-178.0(2)$

$176.8(2)$

$-1.2(2)$

$-4.5(4)$

$177.5(2)$

84.9 (3)

$-93.6(3)$

$1.6(4)$

$-1.5(4)$

$-0.7(4)$

$0.2(4)$

Hydrogen-bond geometry $\left(A,{ }^{\circ}\right)$

\begin{tabular}{lllll}
\hline$D-\mathrm{H} \cdots A$ & $D-\mathrm{H}$ & $\mathrm{H} \cdots A$ & $D \cdots A$ & $D-\mathrm{H} \cdots A$ \\
\hline $\mathrm{N} 2-\mathrm{H} 2 D \cdots \mathrm{N} 6$ & 0.86 & 2.56 & $3.154(3)$ & 128 \\
$\mathrm{~N} 5-\mathrm{H} 5 B^{\cdots}{ }^{\mathrm{N}} 3^{\mathrm{i}}$ & 0.86 & 2.36 & $3.163(3)$ & 156 \\
\hline
\end{tabular}

Symmetry code: (i) $-x+1, y+3 / 2,-z+1 / 2$. 